\title{
Endogenous amino acid pool sizes in mouse eggs and preimplantation embryos
}

\author{
G. A. Schultz, P. L. Kaye, D. J. McKay and M. H. Johnson* \\ Division of Medical Biochemistry, University of Calgary, Calgary, Alberta, Canada T2N 1N4, \\ and ${ }^{*}$ Department of Anatomy, University of Cambridge, Cambridge CB2 3DY, U.K.
}

\begin{abstract}
Summary. The endogenous pool sizes of 17 amino acids were measured directly in samples of mouse eggs, 8-cell embryos and blastocysts by estimation of the fluorescent product of the reaction of o-phthalaldehyde and primary amines. Taurine, glycine, alanine, glutamate and aspartate were detected at high levels. During the transition to the blastocyst, most amino acid pools increased 2-3-fold, but the taurine and glycine pools decreased to about 50 and 10\%, respectively, of the egg value. The amino acid distribution in cumulus masses was similar to that of the egg and embryo samples but different from that of serum.
\end{abstract}

\section{Introduction}

Protein metabolism during preimplantation development is currently the subject of intensive investigation. The technique of two-dimensional electrophoresis, combined with studies utilizing radioactive amino acid precursors, has provided detailed information about changes in protein synthetic patterns and their relationship to development and metabolism (see Van Blerkom, 1977; Levinson, Goodfellow, Vadeboncoeur \& McDevitt, 1978; Braude, 1979). However, because of the difficulties in determining the size of amino acid pools in the limited amount of embryonic material available, qualitative analysis of protein metabolism has only rarely been extended to quantitative assessments (Brinster, Wiebold \& Brunner, 1976; Abreu \& Brinster, 1978; Schultz, Letourneau \& Wassarman, 1979). In the study of Schultz et al. (1979) the pool sizes of leucine and methionine were determined indirectly after their expansion and reaction with radioactive fluorodinitrobenzene (Regier \& Kafatos, 1971).

Direct measurement of the size of endogenous non-expanded amino acid pools is desirable because it provides: (1) a basis for selection of radiolabelled amino acid precursors for synthesis studies, (2) the basis and methodology for accurate assessment of the specific activity of amino acid pools in studies using radioactive precursors, and (3) the means to assess directly changes in pool sizes that may result from conditions affecting amino acid transport, loss and exchange.

Detection of the fluorescent products of the reaction of o-phthalaldehyde and primary amines has allowed resolution and measurement of mixtures of amino acids at levels as low as $10 \mathrm{pmol}$, a factor of sensitivity which is 100 -fold greater than that of the more conventional ninhydrin detection system (Benson \& Hare, 1975). This report presents the results obtained when this technique was applied to the amino acid pools of mouse eggs and preimplantation embryos. 


\section{Materials and Methods}

\section{Animals}

Random bred CFLP and HC-CFLP mice (Hacking and Churchill, Alconbury, U.K.) or ALAS mice (Laboratory Animal Breeding Services, University of Alberta, Edmonton, Alberta, Canada) at 8-12 weeks of age were induced to superovulate as described previously (Holmberg \& Johnson, 1979). Unfertilized eggs were recovered 14-16 h after hCG injection, freed from cumulus cells by exposure to hyaluronidase and washed several times in phosphate-buffered medium containing $0.4 \%(\mathrm{w} / \mathrm{v})$ bovine serum albumin (PB1 + BSA) (Holmberg \& Johnson, 1979). Non-compacted 8-cell embryos and early expanding blastocysts were recovered by flushing oviducts and uteri from mated ALAS animals at 66 and $90 \mathrm{~h}$ after hCG, respectively. Embryos were inspected under the stereomicroscope and any that were abnormal or retarded were discarded. Batches of 1000 or more eggs or 8 -cell embryos and 500 or more blastocysts were collected in $1 \mathrm{ml}$ conical centrifuge tubes and all excess medium drawn off, leaving a final volume of 2-3 $\mu$ l. Part $(5 \mu \mathrm{l})$ of the PB1 + BSA medium was always saved as a control.

\section{Preparation of TCA-soluble amino acids}

Groups of eggs or embryos, cumulus masses or control media, collected as above, were subjected to three freeze-thaw cycles followed by addition of $10 \mu \mathrm{l} 15 \%(\mathrm{v} / \mathrm{v})$ trichloroacetic acid (TCA). The suspensions were mixed and left on ice for $30 \mathrm{~min}$. The TCA-soluble fraction was recovered after centrifugation at $5000 \mathrm{~g}$ for $10 \mathrm{~min}$ at $0^{\circ} \mathrm{C}$. Recovery of $\left[{ }^{35} \mathrm{~S}\right]$ methionine from eggs preincubated in PB $1+$ BSA containing this radioactive precursor was $77.7 \pm 2 \cdot 22 \%$ (mean \pm s.e.m., 5 observations) by this procedure. Similarly, recovery of methionine from extracted PB $1+$ BSA medium containing $100 \mu \mathrm{M}$-methionine was $77.4 \pm 2 \cdot 72 \%$ (mean \pm s.e.m., 4 observations).

Serum was prepared from individual mice bled by cardiac puncture following cervical dislocation. Soluble amino acids were prepared by one of-two methods: (i) addition of $10 \mu$ l $15 \% \mathrm{TCA}$ to $5 \mu \mathrm{l}$ serum and extraction as for mouse eggs and embryos, or (ii) addition of an equal volume of $6 \%$ sulphosalicylic acid before recovery of the soluble fraction after centrifugation.

\section{Amino acid analyses}

To the sample of approximately $15 \mu \mathrm{l}$ acid-soluble extract was added 500 or 1000 pmol norleucine as an internal standard and the total sample was desiccated to dryness. The sample

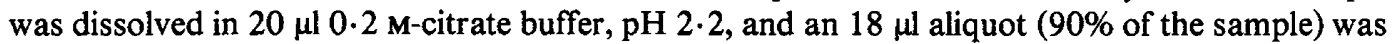
run on a Beckman $121 \mathrm{M}$ Amino Acid Analyzer modified for fluorescence detection using o-phthalaldehyde (Benson \& Hare, 1975) and a Gilson Spectra/Glo Filter Fluorometer essentially as described by Roth \& Hampai (1973). If any of the peaks were off-scale, the remaining $2 \mu \mathrm{l}$ aliquot of sample was also analysed. A Spectra Physics SP4100 computing integrator provided quantitative values which were corrected relative to norleucine for manipulative losses. Quantitative values were also corrected for losses during extraction using the factor of $78 \%$ recovery described above. All quantitative measurements were based on calibration against amino acid standards (Pearce, Rockford, Illinois, U.S.A.) treated identically to the samples. Between 3 and 9 batches of embryos were analysed for each embryonic stage examined.

Abbreviations and single letter codes for amino acids are used as defined by the IUPAC-IUB. Taurine has been abbreviated as Tau. 


\section{Results}

The elution profile of the 16 amino acids which, in addition to glutamine (which elutes between threonine and serine, Text-fig. 1b), are resolved by the procedure used in this study are shown in Text-fig. 1(a). Analysis of a sample of 1626 eggs (Text-figs $1 \mathrm{~b}$ and $1 \mathrm{c}$ ) showed that each of these
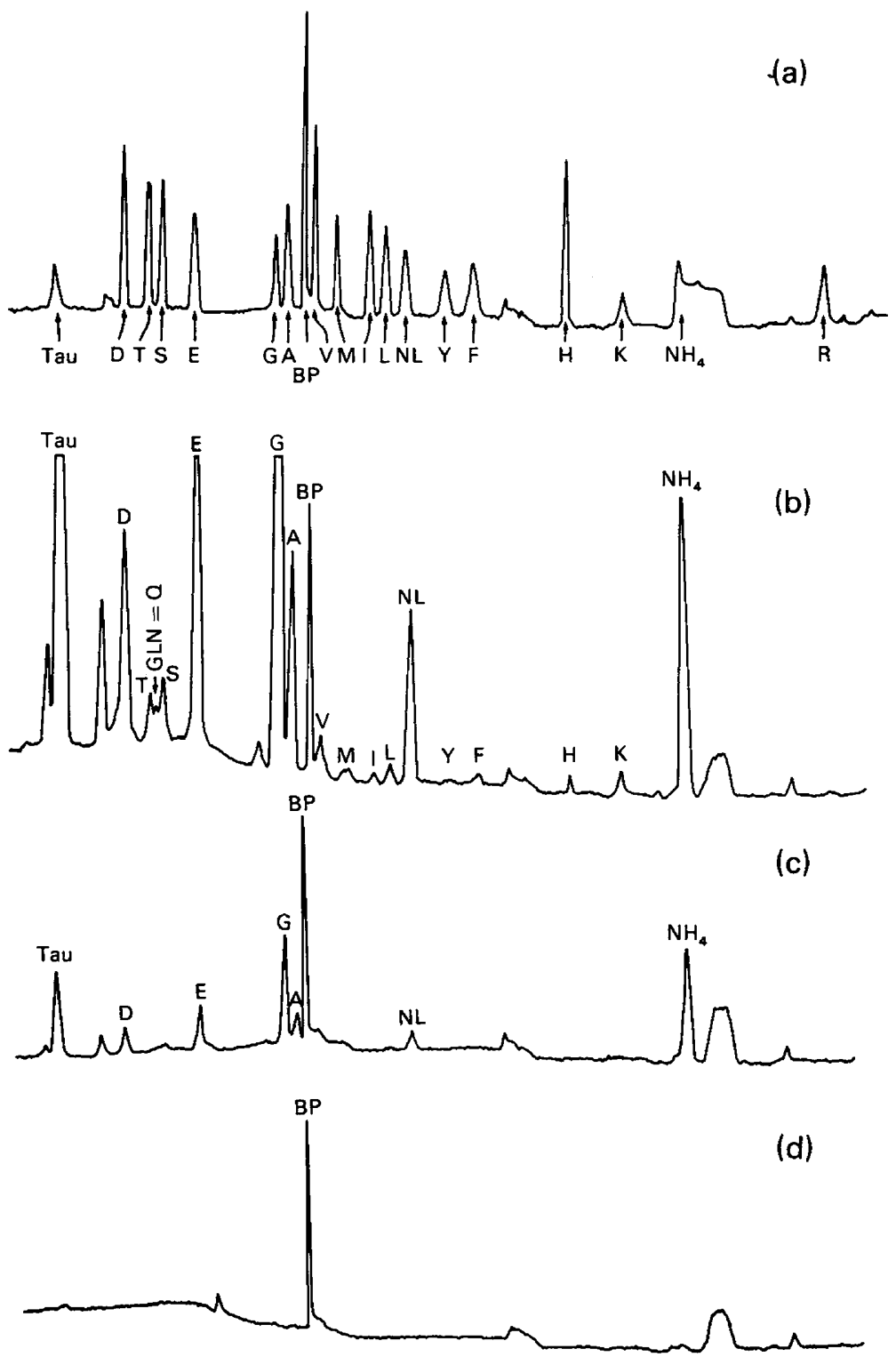

Text-fig. 1. Analysis of TCA-soluble amino acids: (a) 200 pmol amino acid standards; (b) $90 \%$ of a sample from 1626 unfertilized mouse eggs to which 1000 pmol of the norleucine internal standard was added; (c) $10 \%$ of the sample in (b); (d) background of the TCA extracts of $5 \mu \mathrm{l}$ PB1 + BSA medium. $\mathrm{A}=$ alanine; $\mathrm{BP}=$ buffer peak; $\mathrm{D}=$ aspartate; $\mathrm{E}=$ glutamate; $\mathrm{F}=$ phenylalanine; $\mathrm{G}=$ glycine; $\mathrm{H}=$ histidine; $\mathrm{I}=$ isoleucine; $\mathrm{K}=$ lysine; $\mathrm{L}=$ leucine; $\mathbf{M}=$ methionine; $\mathrm{NH}_{4}=$ ammonia; $\mathrm{NL}=$ norleucine; $\mathrm{Q}=$ glutamine; $\mathrm{R}=$ arginine; $\mathrm{S}=$ serine; Tau = taurine; $\mathrm{T}=$ threonine; $\mathrm{V}=$ valine. 
amino acids was present. There was no contamination of the collection medium with any of these amino acids (Text-fig. 1d). Although visible, tyrosine and arginine were below the limit of quantitation of the instrument in samples of up to 2000 eggs.

A summary of the sizes of the amino acid pools in eggs, 8-cell and blastocyst stage embryos is shown in Table 1 . There were high concentrations at each stage of taurine, glycine, alanine and the acidic amino acids, glutamate and aspartate. The 8-cell embryos and blastocysts differed from the eggs in that the pool sizes of most of the amino acids were 2-or 3-fold greater except for those of taurine and glycine which were only about 50 and $10 \%$, respectively, of the egg value by the blastocyst stage. No significant differences in the amounts of particular amino acids were observed between eggs derived from CFLP or ALAS strains.

Table 1. Amino acid pool sizes (mean \pm s.e.m.) of mouse eggs,

8 -cell embryos and blastocysts

\begin{tabular}{lccc}
\hline & \multicolumn{3}{c}{ Amount (fmol/egg or embryo) } \\
\cline { 2 - 4 } \multicolumn{1}{c}{ Amino acid } & $\begin{array}{c}\text { Egg } \\
(n=\mathbf{4}-9)\end{array}$ & $\begin{array}{c}8 \text {-cell } \\
(n=3)\end{array}$ & $\begin{array}{c}\text { Blastocyst } \\
(n=3-5)\end{array}$ \\
\hline Taurine & $6565 \pm 335$ & $1266 \pm 80$ & $3014 \pm 537$ \\
Aspartate & $462 \pm 85$ & $435 \pm 9$ & $749 \pm 119$ \\
Threonine & $124 \pm 8$ & $333 \pm 27$ & $155 \pm 54$ \\
Glutamine & $94 \pm 22$ & $366 \pm 61$ & $348 \pm 108$ \\
Serine & $176 \pm 24$ & $400 \pm 74$ & $331 \pm 70$ \\
Glutamate & $890 \pm 122$ & $1316 \pm 107$ & $1250 \pm 183$ \\
Glycine & $3954 \pm 430$ & $2039 \pm 78$ & $335 \pm 57$ \\
Alanine & $592 \pm 66$ & $565 \pm 9$ & $349 \pm 58$ \\
Valine & $73 \pm 7$ & $301 \pm 82$ & $146 \pm 7$ \\
Methionine & $18 \pm 1$ & $45 \pm 6$ & $94 \pm 30$ \\
Isoleucine & $22 \pm 2$ & $64 \pm 14$ & $79 \pm 11$ \\
Leucine & $52 \pm 5$ & $89 \pm 11$ & $115 \pm 13$ \\
Tyrosine & $<10$ & $<10$ & $119 \pm 28$ \\
Phenylalanine & $25 \pm 2$ & $<10$ & $104 \pm 12$ \\
Histidine & $19 \pm 2$ & $102 \pm 32$ & $205 \pm 76$ \\
Lysine & $85 \pm 6$ & $134 \pm 25$ & $181 \pm 10$ \\
Arginine & $<10$ & $<10$ & $116 \pm 14$ \\
\hline
\end{tabular}

The changes in the absolute values of pool sizes were reflected in changes in the percentage distribution of amino acids within the total pool (Table 2). Table 2 also shows that whilst the distributions of amino acids in eggs, embryos and cumulus masses were similar, all were quite different from that in serum.

\section{Discussion}

The results obtained by application of this technique to a direct analysis of amino acids pools in mouse eggs and early embryos suggest that the approach is both reliable and repeatable. The validity of the results using the technique is verified by comparison of the data obtained for mouse serum (Table 2) with published values (Altman \& Dittmer, 1974). Both show lysine, alanine and glutamine as the predominant amino acids. Further confirmation has been obtained by the analysis of sea-urchin ( $S$. purpuratus) eggs and embryos (unpublished): the results show a very high glycine concentration and similar distribution of amino acids to that reported by Fry \& Gross (1970) who used a different technique. Comparison of serum samples extracted with TCA or sulphosalicylic acid showed that all amino acid concentrations were in the same range by either procedure except for glutamine and lysine which were $40 \%$ lower in sulphosalicylic acid extracts. 
Table 2. Relative proportions of free amino acids in mouse eggs, embryos, cumulus masses and serum

\begin{tabular}{lccccr}
\hline & \multicolumn{5}{c}{ Percentage of total (excluding taurine) } \\
\cline { 2 - 6 } Amino acid & Egg* & 8-cell* & Blastocyst* & Cumulus mass $\dagger$ & Serum $\ddagger$ \\
\hline Asp & 7.0 & 7.0 & 16.0 & 6.3 & 0.8 \\
Thr & 1.9 & 5.4 & 3.3 & 4.7 & 7.7 \\
Gin & 1.4 & 5.9 & 7.4 & 3.3 & 20.0 \\
Ser & 2.7 & 6.5 & 7.1 & 5.9 & 5.1 \\
Glu & 13.5 & 21.3 & 26.7 & 29.3 & 3.3 \\
Gly & 60.0 & 33.0 & 7.2 & 25.6 & 7.2 \\
Ala & 9.0 & 9.1 & 7.5 & 15.6 & 11.9 \\
Val & 1.1 & 4.9 & 3.1 & 0.8 & 6.2 \\
Met & 0.3 & 0.7 & 2.0 & 0.3 & 1.9 \\
Ileu & 0.3 & 1.0 & 1.7 & 0.3 & 3.0 \\
Leu & 0.8 & 1.4 & 2.5 & 0.9 & 4.7 \\
Tyr & Trace & Trace & 2.5 & 1.2 & 2.3 \\
Phe & 0.4 & Trace & 2.2 & 1.3 & 2.3 \\
His & 0.3 & 1.7 & 4.4 & 1.5 & 1.9 \\
Lys & 1.3 & 2.2 & 3.9 & 1.3 & 14.8 \\
Arg & Trace & Trace & 2.5 & 1.6 & 6.9 \\
\hline
\end{tabular}

* Egg and embryo values calculated from data in Table 1.

† Based on 3 determinations, each on 5 cumulus masses containing 56,64 and 72 eggs, respectively. The egg contribution to total amino acid concentration was negligible.

$\ddagger$ Based on analysis of serum samples of 3 different mice.

The high concentration of taurine in eggs is remarkable, especially in view of the apparent metabolic inertness of this sulphoamino acid. As yet, no role for taurine in embryonic metabolism has been described. Similarly, there seems no obvious reason for glycine, aspartate or glutamate to be at such high concentrations in eggs, although aspartate and glutamate do have important roles in intermediary metabolism via transamination and the tricarboxylic acid pathway. The synthesis by early cleaving embryos of glutamate, aspartate and alanine from lactate and pyruvate has been reported, but alanine synthesis is reduced relatively from the 8-cell stage onwards, which may account for the relative fall in total alanine at the blastocyst stage (Quinn \& Wales, 1973). The predominance of glycine, aspartate and glutamate in eggs and early cleavage stages of the rabbit embryo has been reported by Petzoldt, Briel, Gottschewski \& Neuhoff (1973).

No equivalent estimates of the size of endogenous amino acid pools in mouse eggs and embryos have been reported previously. When mouse eggs were incubated in medium containing $25 \mu \mathrm{M}$-methionine for $5 \mathrm{~h}$, Schultz, La Marca \& Wassarman (1978) estimated the expanded pool size to be $74 \mathrm{fmol} / \mathrm{egg}$ by use of the radioactive fluorodinitrobenzene method of Regier \& Kafatos (1971). Using the same method to quantitate the size of the leucine amino acid pool after expansion in medium containing $10 \mathrm{~mm}$-leucine for $4 \mathrm{~h}$, Brinster et al. (1976) obtained a value of $1600 \mathrm{fmol} / \mathrm{egg}$.

Our values for the endogenous non-expanded pool for methionine and leucine are, as expected, much lower at $18 \pm 1$ and $52 \pm 5 \mathrm{fmol} / \mathrm{egg}$, respectively. That intercellular pools can be expanded greatly by incubation in media containing high concentrations of exogenous amino acid is a well documented phenomenon. When eggs were incubated in $100 \mu \mathrm{M}$-methionine for $1 \mathrm{~h}$ at $37^{\circ} \mathrm{C}$, the methionine pool was expanded to $248 \pm 30 \mathrm{fmol} / \mathrm{egg}$ (unpublished). However, there was a simultaneous reduction in the pool size of a number of other neutral amino acids (isoleucine, leucine, phenylalarine) known to be transported through exchange by the same system (Christensen \& Handlogten, 1979; Holmberg \& Johnson, 1979). Applications of the method described in this paper should allow accurate measurement of the size of expanded 
pools, and thence determination of whether the specific activity of an expanded pool actually does equilibrate with that of the external medium when radiolabelled precursors are employed under conditions of pool expansion. At the same time. alterations in other pools affected by exchange can be monitored. Such experiments are in progress.

The changing profile of pool sizes as the egg develops to the blastocyst stage is relevant to a number of factors. First, the general increase in pool sizes of most of the amino acids (with the exception of taurine and glycine) which occurs at the 8-cell stage, precedes the increase in protein metabolism which has been observed by many investigators to accompany the morula-blastocyst transition. Second, a number of workers have shown that mouse embryos can be cultured through the preimplantation stages without provision of exogenous amino acids in the medium, and that the protein content of the embryos is more or less constant during this period. These observations suggest that the embryo is capable of relying on internal stores and catabolism to supply amino acid requirements. Third, it can be expected that fluids of the mouse reproductive tract contain significant quantities of amino acids, as do those of the rabbit (Jaszczak, Hafez, Moghissi \& Kurrie, 1972) and sheep (Wales, 1973), and it is likely that these serve as sources of amino acids for egg and embryo transport and exchange systems during development in vivo. The amino acid distributions in the mouse egg and blastocyst (Table 2) bear some similarity to those of tubal and uterine horn fluids measured in the ewe (Wales, 1973), supporting the view that the amino acid pools of the egg in the cumulus mass, and of the embryos in the genital tract, are to some extent controlled by the maternal environment. Finally, the distribution of amino acids in the total amino acid pool bears no relationship to the amino acid composition of mouse oocyte proteins reported by Bleil \& Wassarman (1980). The same situation has been observed for sea urchin embryos (Fry \& Gross, 1970).

The amino acid pool sizes reported here have immediate application to studies of protein synthesis because they suggest that amino acids such as methionine, leucine, isoleucine and phenylalanine, which have small endogenous pools, should be the most useful radioactive precursors to employ in such studies. Furthermore, provided the specific radioactivity of the amino acid is high and the amount of amino acid added exogenously is small in comparison to the pool (to minimize exchange), it may be possible to use these results to calculate internal specific radioactivity without having to measure the internal pool.

We thank Dr E. H. Peters for advice and J. Fleetham and R. Buckley for technical assistance. This work was supported by an operating grant from the M.R.C. (Canada) to G.A.S. and from the M.R.C. (U.K.) and Ford Foundation to M.H.J. P.L.K. is a recipient of a C. J. Martin Fellowship from the N.H.M.R.C. (Australia).

\section{References}

Abreu, S.L. \& Brinster, R.L. (1978) Synthesis of tubulin and actin during the preimplantation development of the mouse. Expl Cell Res. 114, 135-141.

Altman, P.L. \& Dittmer, D.S. (Eds) (1974) Biology Data Book, 2nd edn, vol. 2, p. 1810. Federation of American Societies for Experimental Biology, Bethesda, Maryland.

Benson, J.R. \& Hare, P.E. (1975) o-Phthalaldehyde: fluorogenic detection of primary amines in the picomole range. Comparison with fluorescamine and ninhydrin. Proc. natn. Acad. Sci. U.S.A 72, 619622.

Bleil, J.D. \& Wassarman, P.M. (1980) Structure and function of the zona pellucida: Identification and characterization of the proteins of the mouse oocyte's zona pellucida. Devl Biol. 76, 185-202.
Braude, P.R. (1979) Control of protein synthesis during blastocyst formation in the mouse. Devl Biol. 68, $440-452$.

Brinster, R.L., Wiebold, J.L. \& Brunner, S. (1976) Protein metabolism in preimplantated mouse ova. Devl Biol. 51, 215-224.

Christensen, H.N. \& Handlogten, M.E. (1979) Interac tion between parallel transport systems examined with tryptophan and related amino acids. J. Neural Trans., Suppl. 15, 1-13.

Fry, B.J. \& Gross, P.R. (1970) Patterns and rates of protein synthesis in sea urchin embryos. II. The calculation of absolute rates. Devl Biol. 21, 125-146.

Holmberg, S.R.M. \& Johnson, M.H. (1979) Amino acid transport in the unfertilized and fertilized mouse egg. J. Reprod. Fert. 56, 223-231. 
Jaszczak, S., Hafez, E.S.E., Moghissi, K.S. \& Kurrie, D.A. (1972) Concentration gradients of amino acids between the uterine and blastocoelic fluid in the rabbit. Fert. Steril. 23, 405-409.

Levinson, J., Goodfellow, P., Vadeboncoeur, M. \& McDevitt, H. (1978) Identification of stage-specific polypeptide synthesis during murine preimplantation development. Proc. natn. Acad. Sci. U.S.A. 75, 3332-3336.

Petzoldt, U., Briel, G., Gottschewski, G.H.M. \& Neuhoff, V. (1973) Free amino acids in the early cleavage stages of the rabbit egg. Devl Biol. 31, $38-46$.

Quinn, P. \& Wales, R.G. (1973) Uptake and metabolism of pyruvate and lactate during preimplantation development of the mouse embryo in vitro. $J$. Reprod. Fert. 35, 273-287.

Regier, J.C. \& Kafatos, F.C. (1971) Microtechnique for determining the specific activity of radioactive intracellular leucine and applications to in vivo studies of protein synthesis. J. biol. Chem. 246, 6480-6488.
Roth, M. \& Hampai, A. (1973) Column chromatography of amino acids with fluorescent detection. J. Chromatogr. 83, 353-356.

Schultz, R.M., La Marca, M.J. \& Wassarman, P.M. (1978) Absolute rates of protein synthesis during meiotic maturation of mammalian oocytes in vitro. Proc. natn. Acad. Sci. U.S.A. 75, 4160-4164.

Schultz, R.M., Letourneau, G.E. \& Wassarman, P.M. (1979) Program of early development in the mammal. Changes in patterns and absolute rates of tubulin and total protein synthesis during oogenesis and early embryogenesis in the mouse. Devl Biol. 68, 341-359.

Van Blerkom, J. (1977) Molecular approaches to the study of oocyte maturation and embryonic development. In Immunology of Gametes, pp. 184-206. Eds. M. Edidin \& M. H. Johnson. Cambridge University Press.

Wales, R.G. (1973) The uterus of the ewe. II. Chemical analysis of uterine fluid collected by cannulation. Aust. J. biol. Sci. 26, 947-959.

Received 13 May 1980 